\title{
APPROACH FOR Egg DEFECTS ASSESSMENT USING IMAGE ANALYSIS
}

\section{Tsvetelina Georgieva, Emil Stefanov, Jakhfer Alikhanov, Zhandos Shynybay, Akmaral Kulmakhambetova \& Plamen Daskalov}
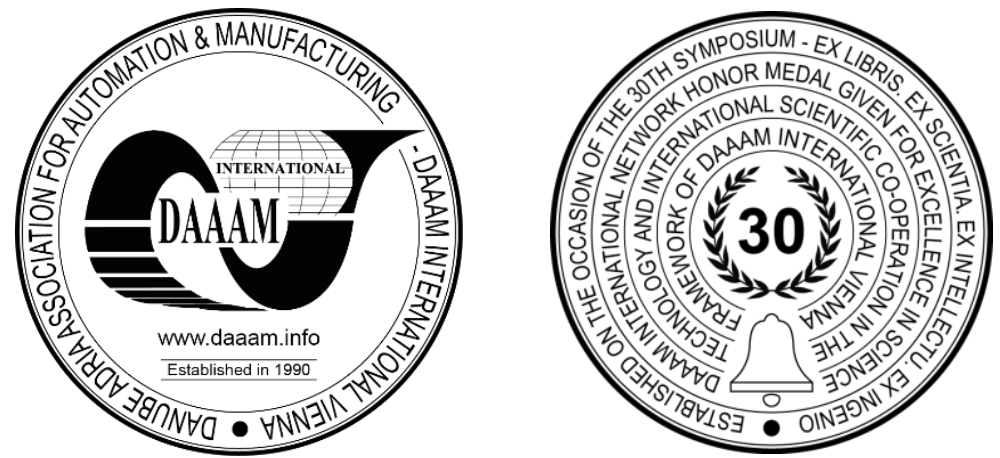

This Publication has to be referred as: Georgieva, T[svetelina]; Stefanov, E[mil]; Alikhanov, J[akhfer]; Shynybay, Z[handos]; Kulmakhambetova, A[kmaral] \& Daskalov, P[lamen] I[vanov] (2019). Approach for Egg Defects Assessment Using Image Analysis, Proceedings of the 30th DAAAM International Symposium, pp.1102-1106, B. Katalinic (Ed.), Published by DAAAM International, ISBN 978-3-902734-22-8, ISSN 1726-9679, Vienna, Austria

DOI: $10.2507 / 30$ th.daaam.proceedings.154

\begin{abstract}
The paper presents approach for egg defects assessment using image analysis. An algorithm for indirect egg defect recognition using image processing is proposed. The values of the egg samples are collected by image processing. HSV and YIQ color spaces are used for egg defects recognition. The paper presents also a developed graphical user interface for recognizing defective eggs in the MATLAB environment, based on computer vision. The interface is modular, which allows upgrade of the procedures and algorithms that are used. The experimental results show that the accuracy with YIQ color model is better than the HSV color model for the purpose of recognition. Accuracy ranges is less than 5\%.
\end{abstract}

Keywords: Egg quality; MATLAB; Computer vision.

\section{Introduction}

Eggs produced from poultry, purchased from private suppliers, are usually of varying mass freshness and quality. Some have defects, reduced storage strength, and some have defects that make them unfit for food. Therefore, eggs have to be assessing with technological procedures, including defect detection and sorting in different quality classes.

- Defect detection

Due to its porous structure, the egg shell appears transparent and allows the eggs to be illuminated. Egg illumination is an observation against light. This method is used when the size and mobility of the air chamber, the state of the yolk and the protein, and egg defects such as cracks and morphological deviation from the normal structure of membranes, in mold, yolk and mobility etc. cannot be established.

- Sorting

Sorting involves the separation of standard and unusual eggs. The process of this technology in standard eggs is divided into homogeneous groups: size, degree of contamination, shell quality and purpose.

Sorting can be done manually or with a machine. Eggs weighing less than 30 grams are not allowed to be sold and is redirected for processing by egg sorting machines. Eggs are divided into the following categories by weight: small, medium, large and very large. 
Sort by purity of shell. Depending on their purity, eggs are divided into:

- Clean - when the surface of the egg is free from visible traces of stool or other contaminants

- Slightly soiled - when dirt occupies $1 / 8$ of the surface of the shell.

- Contaminated - Contamination takes up more than 1/8 of the surface of the shell or in a small area, but very intense.

In all categories, eggs must have a normal structure and thickness, be healthy, clean and undamaged.

Industrially, this can only be done through eggs whose shell is not broken. Cracked and broken eggs are used for processing. The cleanliness of the shell is taken into account, and this is largely dependent on the maintenance of the eggs. Producers may only offer clean or slightly contaminated eggs. Highly contaminated eggs cannot be sold and stored for processing.

Existing egg sorting systems use different type signs of their classification as color, defects, sizes, etc. In these systems for individual quality indicators are used with separate sensors for their own determination. To determine the size of the eggs are uses computer vision [1]. To determine different types of egg defects is used spectrophotometer system [2], [3], [4], [5]. Spectral analysis is also used as a technique for egg quality assessment [6], [7]. The disadvantage of existing commercial systems is their high cost, complexity and maintenance because of the specific equipment they need for their proper functioning.

The paper presents approach for egg defects assessment using image analysis. The paper presents also a developed graphical user interface for recognizing defective eggs in the MATLAB environment, based on computer vision.

\section{Egg defect assessment using image analysis}

\subsection{Egg samples}

Common defects in eggs can be the following types:

- rough cracks (figure 1a) - refers to large cracks and holes that usually lead to a broken membrane of the shell. The incidence of coarse cracks increases with the age of the hen. It varies from 1 to $5 \%$ of total production.

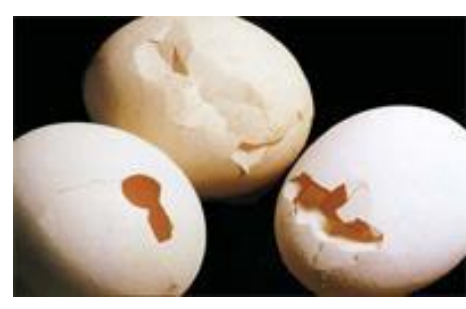

a)

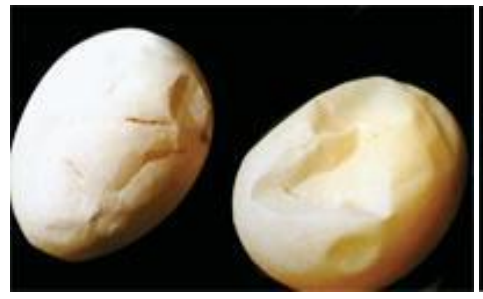

d)

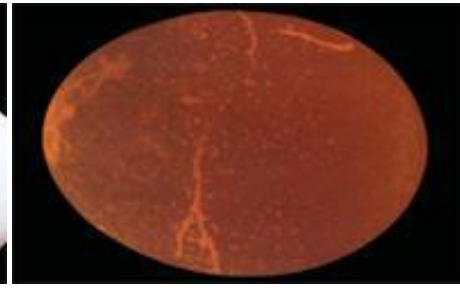

b)

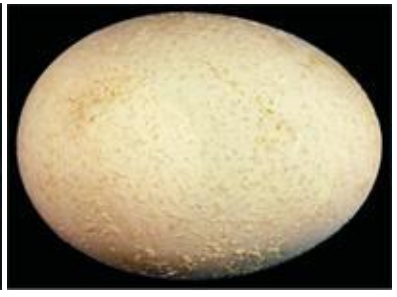

e)

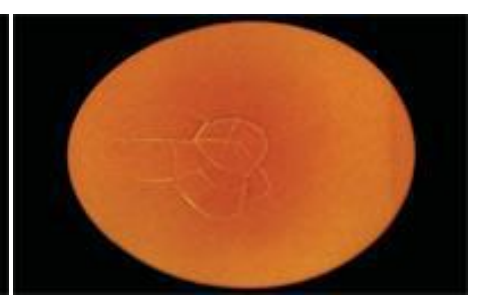

c)

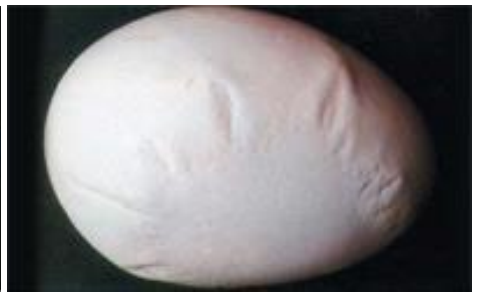

f)

Fig. 1. Egg defects

- very fine cracks (figure 1b), generally crossed long itudinally the length of the casing.

- cracks in the shape of a star (figure 1c) - these are fine cracks, radiating outwards from the central point of impact.

- eggs without shell or very thin shells (figure 1d) - look unattractive and very vulnerable to disability.

- eggs with coarse shells (figure 1e) - refer to eggs with roughly shaped areas, often unevenly distributed on the shell.

- flat eggs - when part of the shell is flattened or cut. Often the adjacent part of the shell is wrinkled.

\subsection{Computer Vision System and Software platform}

Because defects can be assessed visually, it is used computer vision system (figure 2) to capture the image of the eggs. The system consists of a stand with an egg stand and mounted lighting for illumination of the egg from above and below; PC in which it is being recorded the image captured with a color digital camera. The output image is in RGB color model with $640 \times 480$ pixels. 


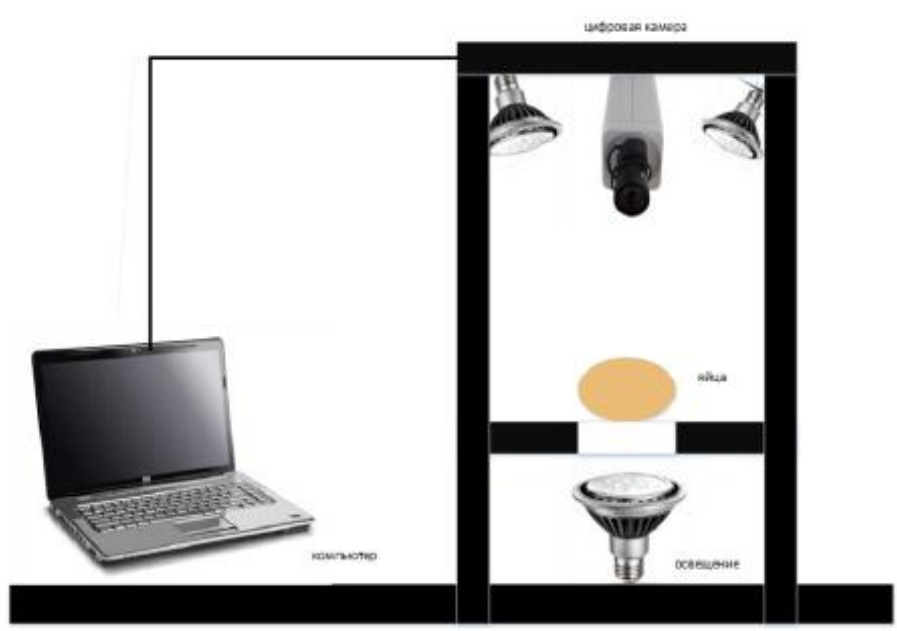

Fig. 2. Computer vision system

The main step is to select an informative color component to be used to detect egg defects. For this purpose three color models were used - Lab, XYZ and HSV.

The software platform was used to develop the interface MATLAB [8]. A part of the graphical user interface is presented on figure 3, which was developed to use to visualize the source image of egg (in RGB pattern), image of egg without background (to be processed only pixels of the egg) and the image of the egg in the three components of each color model.

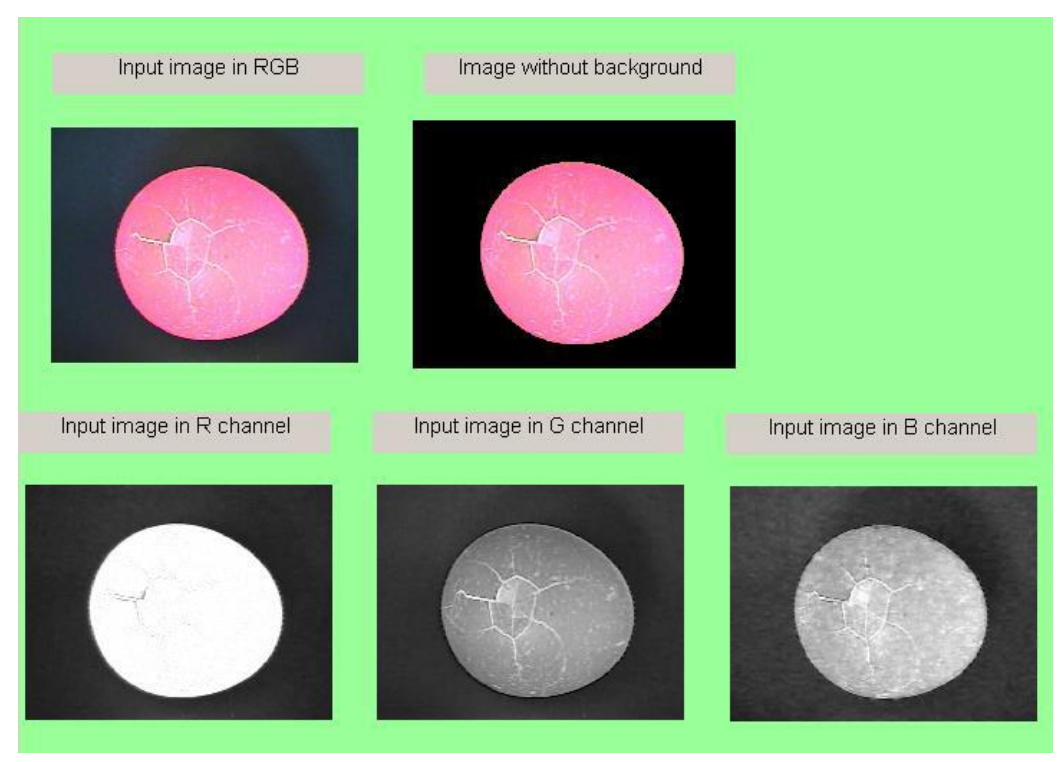

Fig. 3. Graphical user interface for visualizing the output image, background image and the three color model components.

The interface also has an opportunity to visualize the histograms of these components. As an informative, the S component of HSV color model has been evaluated. The defect recognition algorithm for eggs includes the following basic steps:

1. Convert from RGB to HSV color pattern to the egg output image.

2. Remove the pixels from the image background by setting the limits of the $\mathrm{H}$ component.

3. Switch to a black and white image by using a MATLAB built-in function for determining the gray level threshold.

4. Display the contours in the image of the egg.

5. Estimating the egg in one of two possible classes (with or without defects) as a criterion for the absence or presence of defects is the number of contours in the image. If there is one loop - the egg is free of defects, if more than one contour is present - the egg is defective.

This algorithm and the procedures used in its individual steps are embedded in the developed graphical user interface (figure 4). 


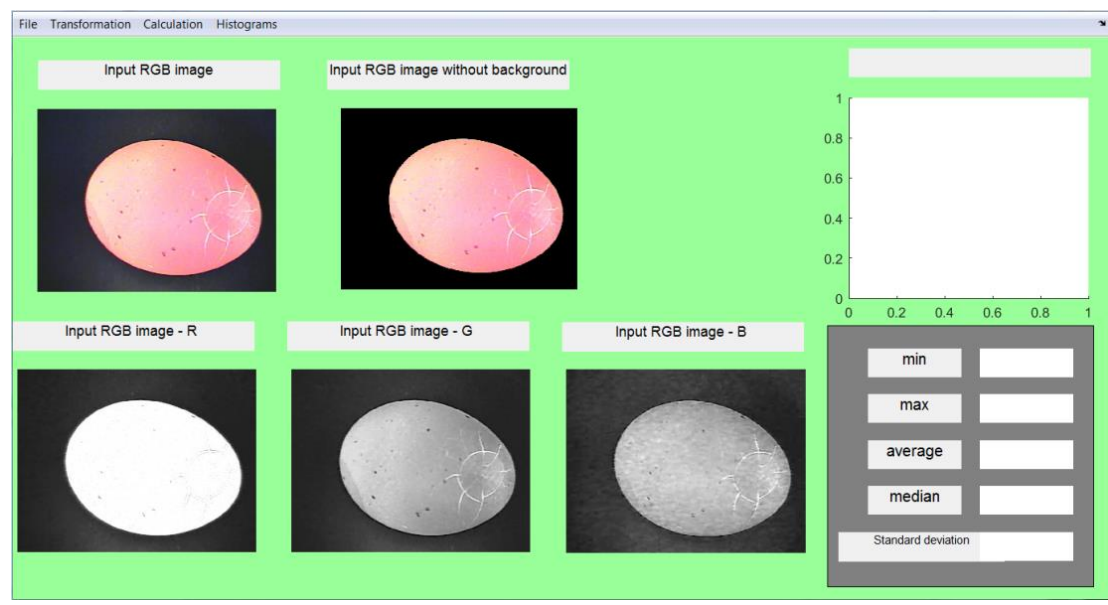

Fig. 4. Graphical user interface for assessment egg defects

\section{Results}

The developed graphical user interface has been tested with 120 eggs, 30 pieces of the four classes s, m, 1 , xl. The test results are presented in table. 1 .

Eggs are appreciated by an expert and by the developed interface. The last column of the table shows the error of percentages from the incorrect recognition of the eggs.

\begin{tabular}{|l|c|c|c|c|c|c|}
\hline $\begin{array}{l}\text { Egg } \\
\text { size }\end{array}$ & \multicolumn{2}{|c|}{ Expert } & \multicolumn{2}{c|}{ Developed software } & \multicolumn{2}{c|}{$\begin{array}{c}\text { Error of percentages from the } \\
\text { incorrect recognition }\end{array}$} \\
\hline & With defect & Without defect & With defect & Without defect & With defect & Without defect \\
\hline S & 13 & 17 & 15 & 15 & $0,6 \%$ & $0,6 \%$ \\
\hline M & 14 & 16 & 16 & 14 & $0,6 \%$ & $0,6 \%$ \\
\hline L & 15 & 15 & 12 & 18 & $0,9 \%$ & $0,6 \%$ \\
\hline XL & 15 & 15 & 22 & 8 & $2,1 \%$ & $2,1 \%$ \\
\hline
\end{tabular}

Table 1. Test results of the developed interface

\section{Conclusion}

The disadvantage of existing commercial systems is their high cost, complexity and maintenance because of the specific equipment they need for their proper functioning. The paper presents an approach for egg defects assessment using image analysis and developed graphical user interface for detecting defective eggs in the MATLAB environment, based on computer vision. The interface is modular, which allows for the upgrade of the procedures and algorithms that are used. It is determined that the S component of HSV color model is informative for egg defects recognition. The accuracy of recognition for the four main qualitative groups of eggs was tested. Accuracy ranges from 0.6 to $2.1 \%$. In the future we will research a methods for improving the classification algorithm.

\section{Acknowledgments}

The study was supported by contract of University of Ruse "Angel Kanchev”, № BG05M2OP001-2.009-0011-C01, " Support for the development of human resources for research and innovation at the University of Ruse "Angel Kanchev". The project is funded with support from the Operational Program " Science and Education for Smart Growth 2014 - 2020" financed by the European Social Fund of the European Union.

\section{References}

[1] Ibrahim, R.; Mohd Zin, Z.; Nadzri, N.; Shamsudin, M. \& Zainudin, M. (2012). Egg's Grade Classification and Dirt Inspection Using Image Processing Techniques, Proceedings of the World Congress on Engineering, Vol. II, July 4 - 6, 2012, London, U.K. 
[2] Lunadei, L.; Ruiz-Garcia, L.; Bodria, L. \& Guidetti, R. (2012). Automatic Identification of Defects on Eggshell Through a Multispectral Vision System, Food Bioprocess Technol, Vol. 5, 3042-3050

[3] Schwagele, F.; POSER, R. \& KROCKEL, L. (2001) Application of low-resolution NMR spectroscopy of intact eggs - Measurement of quality determining physical characteristics, Fleischwirtschaft, 81(10), 103-106.

[4] Chukwuka, O. K.; Okoli, I.; Okeudo, N.; Udedibie, A.; Ogbuewu, I.; Aladi, N.; Iheshiulor, O. \& Omede, A. (2011). Egg quality defects in poultry management and food safety. Asian Journal of Agricultural Research, 5(1), 116.

[5] Öztürk, N. \& Gangal, A. (2014). Eggshell defects detection on white eggs using image processing techniques, 2014 22nd Signal Processing and Communications Applications Conference (SIU), DOI: 10.1109/SIU.2014.6830353

[6] Xie, C. \& He, Y. (2016). External characteristic determination of eggs and cracked eggs identification using spectral signature, Sci Rep., 6, 21130

[7] Sun, D. (2009). Infrared spectroscopy for food quality analysis and control. New York.

[8] https://www.mathworks.com/ 